\title{
Expression of the Intermediate-Filament-Associated Protein Synemin in Chicken Lens Cells
}

\author{
BRUCE L. GRANGER † AND ELIAS LAZARIDES* \\ Division of Biology, California Institute of Technology, Pasadena, California 91125
}

Received 23 April 1984/Accepted 3 July 1984

\begin{abstract}
Synemin, a 230-kilodalton polypeptide component of avian muscle and erythrocyte intermediate filaments, is also found in association with the vimentin filaments of lens tissue. In chicken lens cells, synemin is bound to the core vimentin polymer with the same $180-\mathrm{nm}$ periodicity that it exhibits in erythrocytes. Its solubility properties are characteristic of those of intermediate filaments in general and similar to those of synemin in muscle cells and erythrocytes. Synemin appears at an early stage of lens development and undergoes a dramatic accumulation as the epithelial cells elongate and differentiate into fiber cells. In contrast to synemin in cultured skeletal muscle, lens synemin is not confined to postmitotic, terminally differentiating cells but is present in proliferative cells as well. It is lost from the fibers near the center of the lens, as are many other cellular structures including intermediate filaments. These findings provide new information about the occurrence and expression of avian synemin and new insight regarding its presumptive role as a modulator of intermediatefilament function.
\end{abstract}

The lens offers a unique system for the study of cellular differentiation, since all stages of development are simultaneously present in each lens, the stages are topographically distinct, and no other cell type is present $(1,18,39,40)$. The anterior surface of the lens is covered by a layer of cuboidal epithelial cells; these cells proliferate, elongate, and eventually become the spindle-shaped fiber cells that make up the bulk of the lens. Throughout life, new fibers are continually being added to the periphery, such that the concentric fibers form a centripetal age gradient, with the fibers in the center of the lens being the oldest. In chickens, the elongating epithelial cells form an annular pad at the equator of the lens; the anterior rim of this pad is bordered by a narrow layer of germinal epithelial cells from which all of the new lens cells arise. A period of ca. 2 years elapses between the final mitoses of cells in this bordering layer and movement of these cells through the annular pad to become cortical fiber cells (17). The lens is surrounded by a thin, clear, collagenous capsule within which all of the lens cells are permanently confined.

Many of the molecular changes that accompany lens cell differentiation have been studied extensively $(1,18,39,40)$. As lens cells elongate, there is a dramatic increase in the expression of proteins involved in this morphological change as well as in the accommodation of increased cell volumes and surface areas. Additionally, certain proteins are specifically induced, such as the crystallins that compose most of the protein mass of these cells. Elongating epithelial cells and cortical fibers become packed with intermediate filaments $(2,30,43,46,47)$ composed of a 50 - to 58-kilodalton polypeptide $(2,22,27-29)$ identified as vimentin (46). No other intermediate-filament subunit has been detected in lens cells (46), but we show here that the vimentin filaments in lens cells coexist with the associated protein, synemin.

Synemin is a 230-kilodalton polypeptide that is found in association with certain types of intermediate filaments in a subset of the cells that possess these filaments. Specifically,

\footnotetext{
* Corresponding author.

† Present address: Section of Cell Biology, Yale University School of Medicine, New Haven, CT 06510.
}

avian synemin has been demonstrated to coexist with desmin filaments in smooth and skeletal muscle but not with desmin in mature cardiac muscle $(12,42)$. It is found with vimentin in skeletal-muscle cells and erythrocytes but not with vimentin in other mesenchyme-derived cells $(12,15$, 42). It coexists with the major neurofilament subunit in erythrocytes from young chickens (14) but not with neurofilaments in neurons (42). In this report, we document the presence of synemin in avian lens cells, compare it with the synemins of muscle cells and erythrocytes, monitor its appearance and expression during the genesis and growth of the lens, and examine its postulated role as a tissue-specific cross-linker of intermediate filaments.

\section{MATERIALS AND METHODS}

Polyacrylamide gel electrophoresis. One-dimensional sodium dodecyl sulfate (SDS)-polyacrylamide slab gel electrophoresis and two-dimensional isoelectric focusing-SDSpolyacrylamide gel electrophoresis were performed by the methods of Laemmli (23) and O'Farrell (37), respectively, essentially as described previously (25). Isoelectric-focusing gels contained no detergent. Separating gels contained $12.5 \%$ acrylamide and $0.1 \% N, N^{\prime}$-methylene-bisacrylamide. SDS sample buffer contained $1 \%$ SDS, $125 \mathrm{mM}$ Tris-chloride (pH 6.8), 10\% glycerol, $1 \mathrm{mM} \mathrm{Na}{ }_{2}$ EDTA, $20 \mathrm{mM}$ dithiothreitol, and $0.004 \%$ bromophenol blue.

Sample preparation. Lenses from a month-old White Leghorn chicken were separated into epithelial and fiber fractions with fine forceps and a stereo microscope (Fig. 1). The epithelium was boiled directly in SDS sample buffer. The fibers were further separated into crude cortical and nuclear fractions of approximately equal volume. These were homogenized in a motor-driven Teflon-glass (Potter-Elvehjem) homogenizer in a large volume of cold $140 \mathrm{mM} \mathrm{NaCl}-16 \mathrm{mM}$ Tris-4 mM EDTA (pH 7.8)-0.5 mM phenylmethylsulfonyl fluoride and sedimented for $10 \mathrm{~min}$ at $20,000 \times \mathrm{g}$. The residues were then boiled in SDS sample buffer.

Decapsulated lenses from adult chickens were homogenized as described above in cold $10 \mathrm{mM} \mathrm{N}$-2-hydroxyethylpiperazine- $N^{\prime}$-2-ethanesulfonic acid-5 $\mathrm{mM} \mathrm{MgCl}_{2}-5 \mathrm{mM}$ $\mathrm{NaN}_{3}-2 \mathrm{mM}$ ethylene glycol-bis ( $\beta$-aminoethyl ether)- 
$N, N, N^{\prime}, N^{\prime}$-tetraacetic acid (EGTA)-0.1 mM dithiothreitol$0.5 \mathrm{mM}$ phenylmethylsulfonyl fluoride (Tris base to $\mathrm{pH} 7.0$ at $20^{\circ} \mathrm{C}$ ) (Fig. $\left.2 \mathrm{~A}\right)$. The residue was then extracted with cold $1 \mathrm{M} \mathrm{KCl}-1 \%$ Triton X-100-1 mM dithiothreitol-2 mM EDTA-10 mM Tris-chloride (pH 6.8) for $1 \mathrm{~h}$, rinsed with 2 mM EDTA-10 mM Tris-chloride (pH 6.8), dissolved in saturated urea containing 1\% 2-mercaptoethanol, and loaded onto an isoelectric-focusing gel (Fig. 2C).

Decapsulated lenses were homogenized and washed in cold $10 \mathrm{mM}$ piperazine- $N, N^{\prime}$-bis(2-ethanesulfonic acid)-5 $\mathrm{mM} \mathrm{MgSO}_{4}-1 \mathrm{mM}$ EGTA-0.1 mM phenylmethylsulfonyl fluoride (Tris base to $\mathrm{pH} 7.0$ ) and then incubated in the same solution containing $2 \mathrm{mM} \mathrm{Na} \mathrm{S}_{4} \mathrm{O}_{6}$ for $1 \mathrm{~h}$ at $0^{\circ} \mathrm{C}$. The tetrathionate was washed out, and the cross-linked cell ghosts were quickly extracted with $1 \mathrm{mM}$ ethanolamine (14, 15). The extract was lyophilized, dissolved in saturated urea containing $20 \mathrm{mM}$ dithiothreitol, and loaded on an isoelectric-focusing gel (Fig. 2C).

The amount of starting material used for the samples on the two-dimensional gels was one lens for Fig. 2A and seven lenses for Fig. 2C.

Antisera. Production and characterization of rabbit antichicken gizzard synemin have been described previously $(12,13,15)$. Antisera to the other intermediate-filament proteins have also been described $(11,12,14)$. All were fractionated with ammonium sulfate before use (11). Fluorescein-conjugated goat anti-rabbit immunoglobulin $G$ was purchased from Miles-Yeda, Ltd., Rehovot, Israel.

Immunoelectrophoretic techniques. Polypeptides were

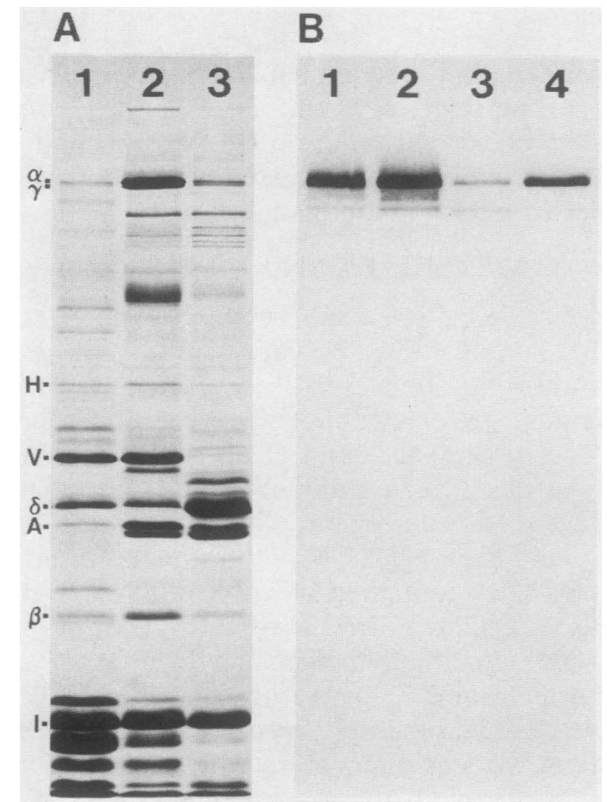

FIG. 1. Immunoblot analysis of synemin in different lens fractions. (A) Coomassie blue-stained gel of chicken lens fractions; (B) antisynemin immunoblot of the same samples, as well as a chicken erythrocyte membrane sample (lane 4), transferred from another gel. Lane 1, whole epithelium (primarily annular pad cells); lanes 2 and 3, physiological salt residues of cortical and nuclear fibers, respectively. Abbreviations: $\alpha$ and $\gamma$, alpha and gamma spectrin (reference 34); H, constitutive 68-kilodalton "heat shock" protein (reference 54); V, vimentin; $\beta$ and $\delta$, beta and delta crystallin, respectively; A, actin; I, major intrinsic protein (MP26) of lens tissue. The polypeptides below $\beta$ are primarily $\alpha$-crystallins and other $\beta$-crystallins. transferred from SDS polyacrylamide slab gels to nitrocellulose for detection with antisynemin and iodinated protein A ("immunoblotting" [10, 52]) as described elsewhere (14a). The autoradiogram in Fig. 1B represents a 1-h exposure without intensification.

Alternatively, antigen detection was performed directly on the gel by immunoautoradiography (4) as previously described (25). The autoradiogram in Fig. 2B represents a 123$h$ exposure with an intensifying screen.

Immunofluorescence. Immunofluorescence was performed essentially as described previously (14a). Chicken embryos were immersed in fixative [piperazine- $N, N^{\prime}$-bis(2-ethanesulfonic acid)-buffered saline with $2 \%$ formaldehyde] for 5 to 10 min, embedded and frozen in Tissue-Tek II O.C.T. compound (Miles Laboratories, Inc., Naperville, Ill.), cut into 4to 6- $\mu \mathrm{m}$ sections, thawed onto glass cover slips, and immersed in fixative for 1 to 2 min either immediately or after air drying. Subsequent washing and antibody incubations were performed in Tris-buffered saline containing $0.5 \%$ Triton X-100. Antisera were diluted 100- to 200-fold. Double immunofluorescence, with rhodamine-conjugated antivimentin, was performed as previously described (11). For photography, cover slips were mounted in $140 \mathrm{mM} \mathrm{NaCl}-$ $2.5 \%$ DABCO [1,4-diazabicyclo-(2,2,2)-octane; Sigma Chemical Co., St. Louis, Mo.]-10 mM Tris-chloride (pH 8.5) to inhibit photobleaching (20).

Electron microscopy. Antibody decoration and rotary shadowing were performed as previously described (13). For negative staining, chicken lens epithelial cells and cortical fibers were homogenized in Tris-buffered saline as described above and then were sedimented and suspended twice in this buffer. The final suspension was incubated with 0.03 volume of antisynemin at $0^{\circ} \mathrm{C}$ for $30 \mathrm{~min}$, sedimented through Trisbuffered saline, applied to a parlodion-covered grid, and negatively stained with aqueous $2 \%$ uranyl acetate.

\section{RESULTS}

Identification of lens synemin. Antibodies raised against chicken smooth-muscle synemin reacted specifically with a 230-kilodalton polypeptide in chicken lens cells, as assayed by immunoblotting (Fig. 1). This polypeptide was present in the epithelium and cortical fibers but nearly absent from the nuclear fibers (Fig. 1A and B, lanes 1 through 3). Its abundance in these fractions correlated with that of vimentin, which was also present in the epithelium and cortical fibers and lacking in the nuclear fibers (Fig. 1A). This is consistent with previous demonstrations of the absence of intermediate filaments and vimentin from nuclear fibers $(2$, $6 a, 8,44,46)$. The cross-reactive lens polypeptide had the same electrophoretic mobility as chicken erythrocyte synemin (Fig. 1B, lane 4), which has been shown to comigrate with muscle synemin (15).

Two-dimensional immunoautoradiography confirmed that the cross-reactive polypeptide was indeed synemin. A twodimensional gel of lens fiber cytoskeletons is shown in Fig. $2 \mathrm{~A}$, and the corresponding antisynemin immunoautoradiogram is shown in Fig. 2B. This technique allowed visualization of proteolytic degradation products that were generated by endogenous proteases during sample preparation and revealed that the pattern for the lens antigen (Fig. 2B) was the same as that for both muscle synemin and erythrocyte synemin (15). The characteristic S-shaped string of fragments in this fortuitous form of peptide mapping (15) terminated in a relatively protease-resistant 34-kilodalton peptide (Fig. 2, arrows). Comigration of the immunoreactive fragments was confirmed by running lens and erythrocyte sam- 

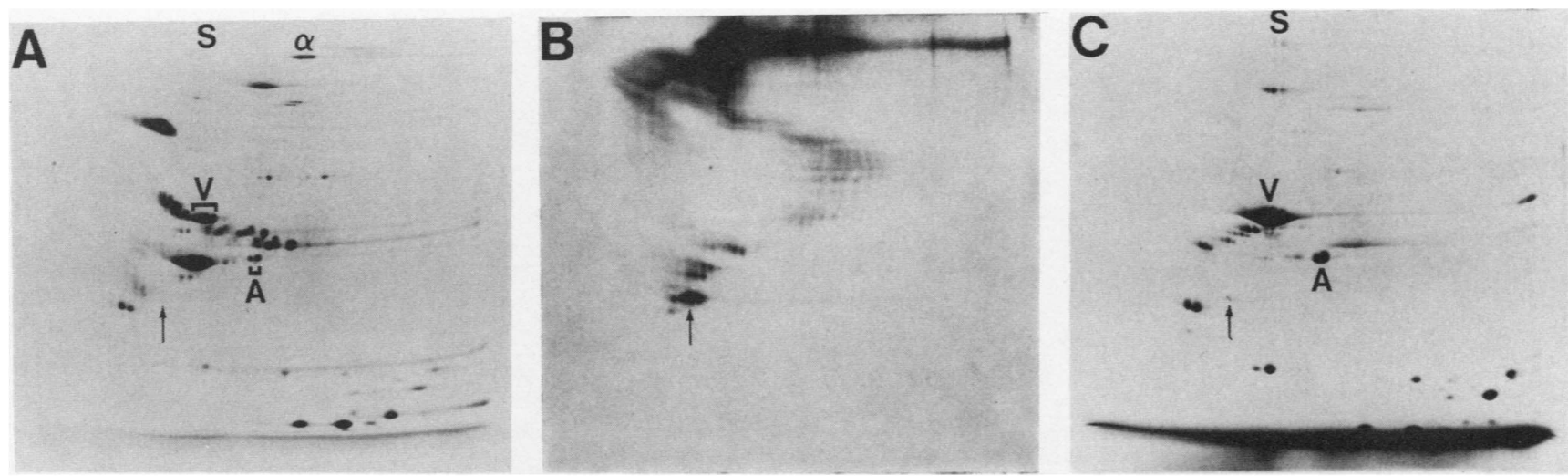

FIG. 2. Two-dimensional gels and an immunoautoradiogram of lens fiber cytoskeletons and intermediate filaments. (A) Coomassie bluestained gel of fibers extracted with high salt and nonionic detergent. (B) Corresponding synemin immunoautoradiogram, overexposed to reveal the degradation products of synemin. (C) Low-salt extract of oxidatively cross-linked fiber residues enriched in the intermediatefilament proteins vimentin (V) and synemin (S). Arrows point to a protease-resistant fragment of synemin. The two variants of actin (A) comigrate exactly with the $\beta$ and $\gamma$ actin variants of chicken erythrocytes (cf. references 8 and 46). Isoelectric focusing was performed from right to left (pH gradient from 4 [left] to 7).

ples (containing equivalent amounts of synemin) together on the same two-dimensional gel and then processing the gel for immunoautoradiography (data not shown). This antiserum did not detect a corresponding polypeptide in rat or bovine lens samples (data not shown); if an analog of synemin indeed exists in mammalian tissues, it must react poorly, if at all, with these antibodies, since muscle cells are similarly unreactive (12).

Lens intermediate filaments and plasma membranes remain associated with each other when the cells are disrupted by osmotic lysis or homogenization but can be separated by denaturing agents or slightly alkaline solutions of very low ionic strength (45). A similar relationship pertains to avian erythrocyte intermediate filaments and plasma membranes (15). In the latter case, a relatively pure preparation of intermediate-filament proteins can be obtained by treating the membrane-filament complexes with low salt and harvesting the extract $(14,15)$. However, application of this procedure to lens cells revealed that the extraction of the intermediate-filament proteins was not nearly as selective. A two-dimensional gel of such an extract (data not shown) was qualitatively similar to the gel shown in Fig. 2A but with relatively more actin and crystallin polypeptides. However, if osmotically lysed lens cells were first oxidatively crosslinked (34), many of these polypeptides became unextractable (presumably through disulfide bonding), and the low-salt extract consequently was greatly enriched for intermediatefilament proteins (Fig. 2C). Vimentin was the major component of the extract, as expected, and synemin was present in proportionate amounts. Lens intermediate filaments were also found to be somewhat more susceptible to extraction by moderately low-ionic-strength (10 to $20 \mathrm{mM})$ solutions containing divalent cation chelators than are erythrocyte intermediate filaments (compare references 15, 34, and this paper), suggesting slightly different affinities or modes of attachment of the filaments to the membranes.

The mobility of lens synemin in SDS gels was variable. Synemin often migrated as a broad, diffuse band or with a mobility lower than that of muscle and erythrocyte synemin (Fig. 2). Whereas muscle and erythrocyte synemin migrates slightly faster than $\alpha$-spectrin $(14,15)$, lens synemin often migrated more slowly than $\alpha$-spectrin in this study. The molecular basis for this variability has not been determined.
The possiblity that it is due to posttranslational modifications related to aging in the lens seems unlikely in light of the observation that newly synthesized synemin comigrated with the bulk of the synemin, as assayed by immunoprecipitation of synemin from $\left[{ }^{35} \mathrm{~S}\right]$ methionine-labeled lenses (data not shown). Since lens synemin occasionally migrated as a sharp band coincident with erythrocyte synemin, the observed heterogeneity may have been an artifact of sample preparation conditions. Many factors are known to be capable of influencing the mobility of polypeptides on SDS gels, but we have been unable to determine which of them is likely to be responsible for the variable mobility of synemin.

The isoelectric point of lens synemin (Fig. 2A and C) is the same as that of muscle and erythrocyte synemin, which is the same as that of vimentin, ca. $5.3(15,50)$. Lens vimentin exhibits the same multiple isoelectric variants as it does in other cell types $(8,15)$; newly synthesized lens vimentin comigrated with the most basic isoelectric variant (data not shown), in accordance with the posttranslational generation of the more acidic variants through phosphorylation $(9,36)$.

Distribution of synemin in developing lens tissue. To investigate the spatial and temporal appearance of synemin during the formation of the lens, we examined cryotome sections of chicken embryos at different stages of development, by immunofluorescence. Synemin was first detectable at the lumenal side of the invaginated ectodermal cells that make up the lens vesicle (Fig. $3 A$ and $A^{\prime}$ ). As the lens vesicle budded off and began to flatten, synemin began to accumulate preferentially in the cells adjacent to the optic cup (Fig. $3 B$ and $B^{\prime}$ ). There was a dramatic increase in the quantity of synemin upon elongation of these cells to form the fiber cells (Fig. 3C and $C^{\prime}$ ). Synemin was most abundant at the back of the lens, adjacent to the optic cup. In the epithelial cells, synemin was most prevalent at the front, adjacent to the presumptive cornea.

This pattern was maintained as the lens increased in size and attained its lentoid shape (Fig. 3D and $\mathrm{D}^{\prime}$ ). The annular pad, which is evident after 4.5 days of incubation (17), had not yet accumulated much synemin. By 10 days of incubation, though, these elongating epithelial cells were rich in synemin (Fig. 3E and $E^{\prime}$ ). These latter micrographs show the region where the epithelial cells differentiate into the cortical fiber cells and demonstrate the preferential distribution of 

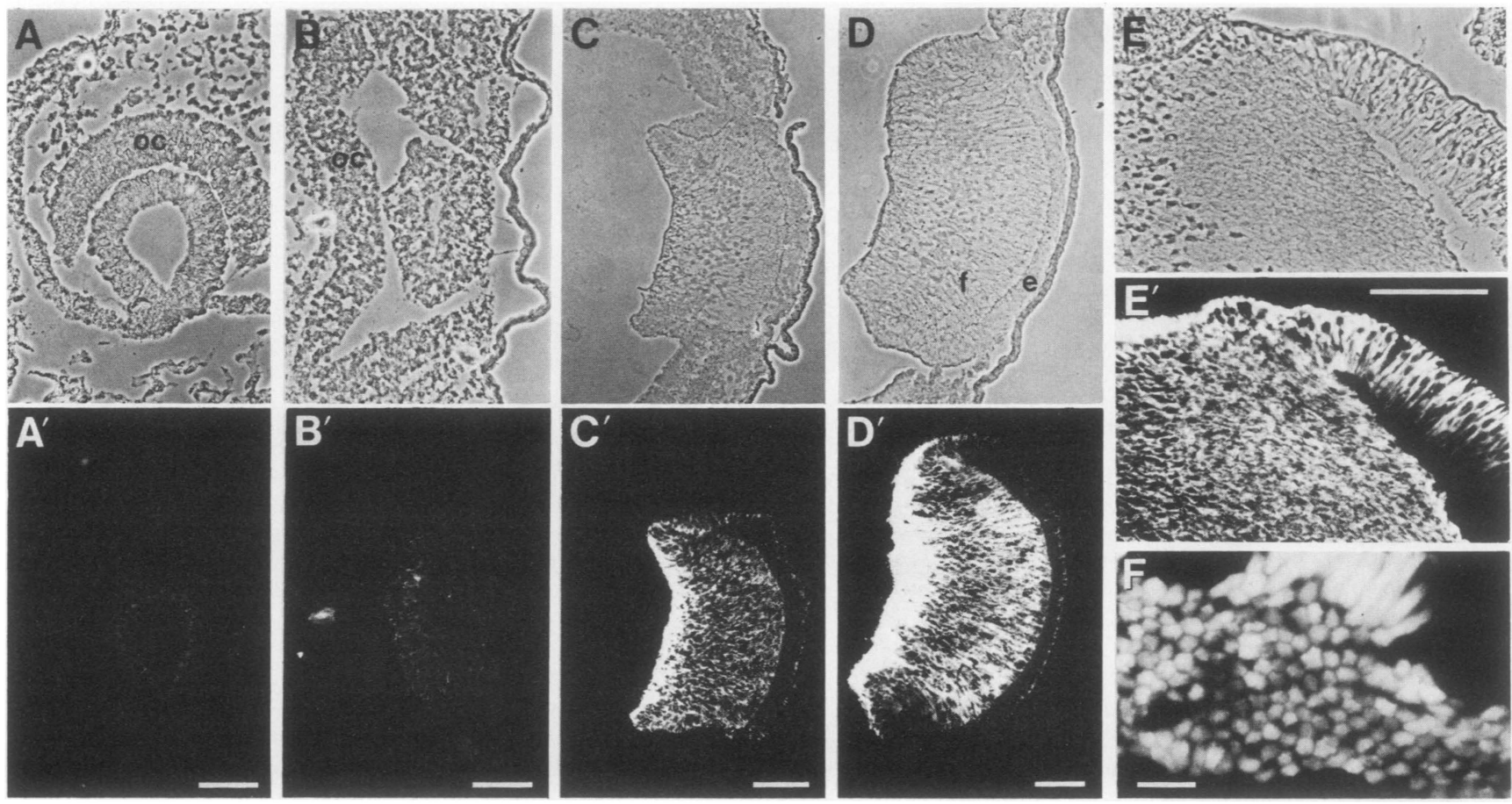

FIG. 3. Immunofluorescence of synemin in lenses of different ages. Phase contrast (A through E) and corresponding fluorescence (A' through $E^{\prime}$ ) micrographs of cryotome sections of embryonic chicken lenses at the following developmental stages (according to references 16 and 38): 16, 18, 23, 25, 36 (A through E, respectively). These correspond to the following periods (in days) of egg incubation: 2 to $2.5,2.5$ to 3 , 3.5 to $4,4.5$, and 10, respectively. (F) Fluorescence micrograph of a portion of the annular pad of an adult chicken lens, with most of the elongating cells cut perpendicular to their long axes. Abbreviations: oc, optic cup; $f$, lens fiber cells; $e$, lens epithelium. The layer of elongating epithelial cells (right) is artifactually separated from the fiber cells in panel E. Note nuclei in panel E (left side). Bars, $0.1 \mathrm{~mm}$ in panels A through $\mathrm{E} ; 20 \mu \mathrm{m}$ in panel $\mathrm{F}$

synemin along the outer, anterior side of the columnar epithelial cells. The elongating epithelial cells of the annular pad in the adult chicken contained synemin throughout their cytoplasm (Fig. 3F). Other sections of adult lens tissue suggested that all of the epithelial cells contained synemin and that, in the fiber cells, most of the synemin was confined to (and evenly distributed within) the cortical fibers, with virtually no synemin detectable in the nuclear fibers (data not shown).

Visualization of both synemin and vimentin in the same sections by double immunofluorescence revealed identical distributions of these two antigens (data not shown). The only apparent differences involved the relative intensities of the fluorescence in different parts of the adult lens; the synemin fluorescence of the epithelium and cortical fibers was of similar intensity, but the vimentin fluorescence of the epithelium was noticeably more intense than that of the cortical fibers. Also, there was a relatively sharp boundary between the nuclear and cortical fibers revealed by the vimentin antibodies but a gradual transition when the fibers were examined with the synemin antibodies. These differences might reflect changes in antigenicity (due to masking or degradation, for example) rather than changes in the absolute quantities of vimentin and synemin. There also seemed to be an earlier appearance of vimentin than synemin in the very early lens rudiments, but the qualitative nature of immunofluorescence precludes the conclusion that lens vimentin exists at some stage of lens cell differentiation without synemin.

Antibodies that react with desmin, epidermal keratins, neurofilament proteins, and glial-filament protein did not detect any of these intermediate-filament antigens in lens cells by immunofluorescence or immunoblotting, nor were these polypeptides evident on two-dimensional gels of lens fiber cytoskeletons or $\left[{ }^{35} \mathrm{~S}\right]$ methionine-labeled lens epithelial-cell proteins (data not shown). Similar conclusions regarding the exclusivity of vimentin in mammalian lens cells have been reached by Ramaekers et al. (46). However, these data do not rule out the presence of nonepidermal keratins or very minor amounts of these other antigens in chicken lens cells or the presence of any of these antigens at stages of development that we have not examined (18a).

Immunoelectron microscopy. The spatial relationship of synemin to the core vimentin filament has previously been investigated in sonically disrupted erythrocytes by using immunoelectron microscopy (13). This technique was adapted to lens cells by using cryotomy as a starting point for the display of the filaments. Frozen sections of embedded chicken lenses were thawed onto glass cover slips and then either air dried (to promote adhesion of the section to the cover slip) or immersed in a physiological buffer with or without fixative. Portions of the lens sections were often lost, but an adherent residue of filaments and membranes usually remained in such regions. These structures could then be shadowed with platinum, and the resulting replicas could be examined by transmission electron microscopy. Curiously, the lens sections on the cover slips were also encircled by free filaments; an annulus of filaments several millimeters wide usually surrounded each section. The means by which these filaments were translocated from the 

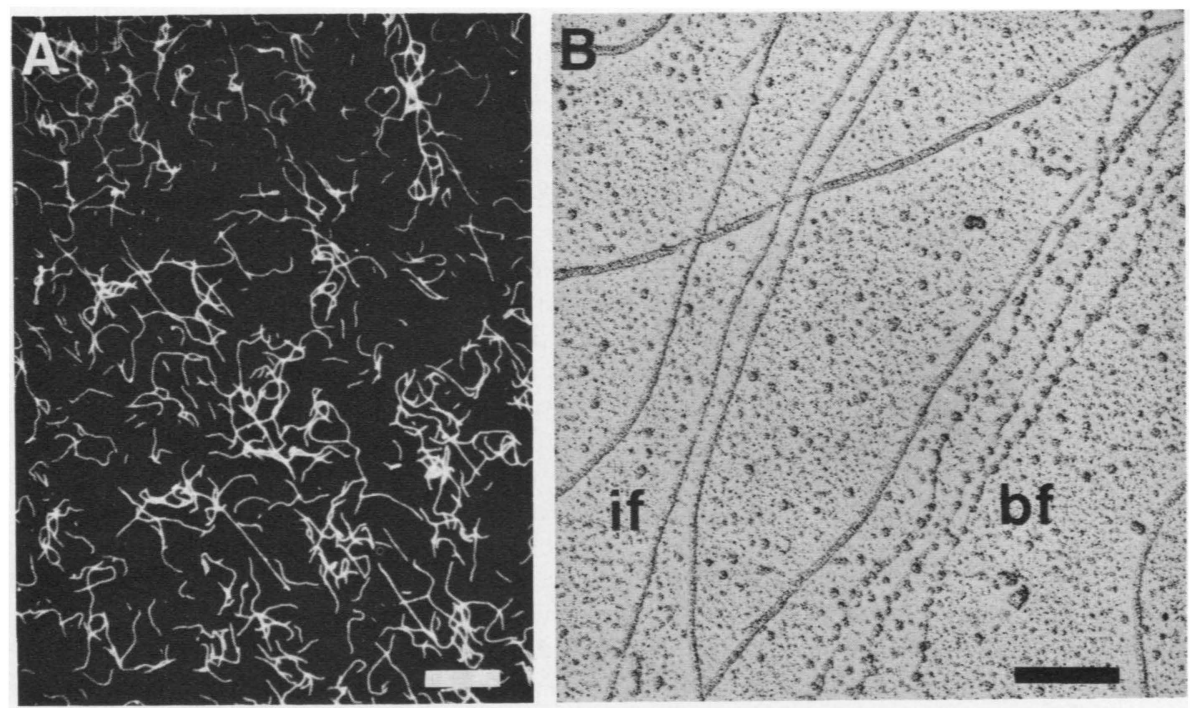

FIG. 4. Lens filaments adhering to a glass substrate. (A) Vimentin immunofluorescence micrograph of intermediate filaments that originate from lens cryosections and become deposited on the cover slip. Bar, $10 \mu \mathrm{m}$. (B) Transmission electron micrograph of rotaryshadowed filaments prepared as in panel A, except that the synemin preimmune serum was used. This nonimmune serum does not alter the appearance of the intermediate filaments (if) or beaded filaments (bf). Bar, $200 \mathrm{~nm}$.

lens section to the surrounding cover slip was not determined, but this phenomenon was reproducible and useful for generating a display of native filaments on a flat substrate.

Such an area after processing for vimentin immunofluorescence is shown in Fig. 4A. The intermediate filaments were usually oriented randomly, but occasionally the longest filaments in a given field appeared straight and parallel to each other. An electron micrograph of a rotary-shadowed replica of such a preparation, with the smooth-contoured intermediate filaments and abundant beaded filaments adhering to the glass substrate, is shown in Fig. 4. (The beads of the beaded filaments are probably crystallin polypeptide complexes; the backbone of these filaments has not been identified, but it does not appear to be DNA, RNA, or actin $[19,30]$.) This sample was actually preincubated with normal rabbit serum (synemin preimmune serum), but the image was indistinguishable from those of untreated samples.

If such preparations were incubated with antibodies to synemin before shadowing, the intermediate filaments were specifically decorated (Fig. 5A, B, and C). This decoration was similar to that demonstrated previously for chicken erythrocyte intermediate filaments, with periodically spaced foci of antibodies along the lengths of the filaments (13). Adjacent foci on individual filaments occasionally appeared to be linked to one another (Fig. 5B and C). These antibodies also demonstrated cross bridging of adjacent filaments, linkage of intersecting filaments, and lateral registry of synemin foci in bundles of filaments (data not shown); such images were virtually identical to those seen in avian erythrocytes (13) and are indicative of a filament-cross-linking,
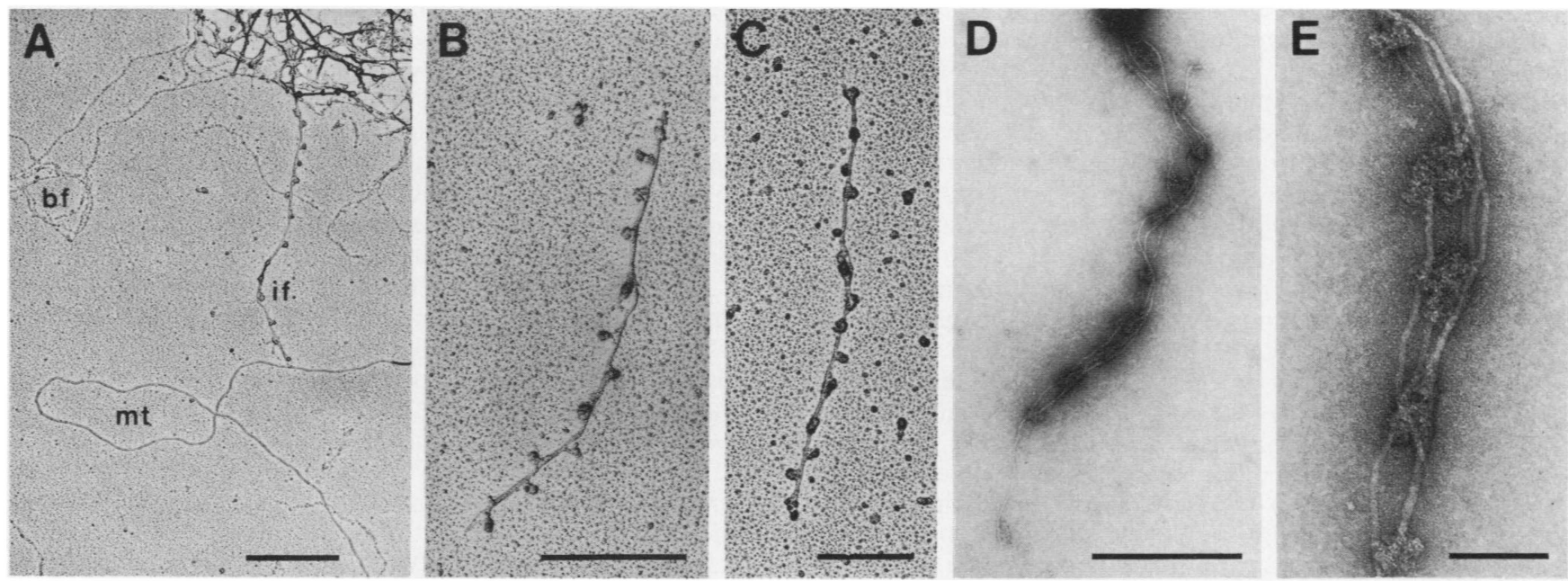

FIG. 5. Synemin decoration of lens intermediate filaments. (A, B, C) Micrographs of rotary-shadowed filaments decorated with antibodies specific for synemin. Beaded filaments (bf) and an undecorated filament, probably a microtubule (mt), are evident in panel A. (D, E) Bundle of intermediate filaments incubated with antisynemin before negative staining. Similar periodicity in the synemin decoration is apparent by both techniques. Bars, $1,000 \mathrm{~nm}$ in panel $\mathrm{A}, 500 \mathrm{~nm}$ in panels B through $\mathrm{D}$, and $100 \mathrm{~nm}$ in panel $\mathrm{E}$. 
network-stabilizing role for synemin in lens cells as well as in erythrocytes (see below). Microtubules and beaded filaments were not decorated with these antibodies, nor were they decorated with antibodies to vimentin, which, as in chicken erythrocytes (13), uniformly coated the intermediate filaments (data not shown).

Antisynemin binding was also examined by negative staining. Lens homogenates were incubated with antisynemin, applied to a parlodion-covered grid, and negatively stained with uranyl acetate (Fig. 5D and E). By this method of analysis, a bundle of filaments could be seen to exhibit periodically spaced clusters of antibodies. If no antibodies were applied, only smooth, uniform filaments were seen (data not shown; see references 29 and 30). The registry of the filaments in this bundle also exemplifies the presumptive cross-linking role of synemin.

To determine the linear periodicity of lens synemin, we measured 300 nodes on 18 rotary-shadowed filaments. The average spacing was $180 \mathrm{~nm}$, which is the same as the synemin spacing on adult chicken erythrocyte intermediate filaments (13). The average spacing on individual lens filaments, however, ranged from 130 to $230 \mathrm{~nm}$. Whether these spacing differences were due to such factors as the age of the chicken, the age of the cells from which the filaments came, or simply variations in preparative procedures (such as the presence or absence of fixatives or nonionic detergents) has not been determined. In contrast, a clear difference in the periodicity of synemin between embryonic and adult erythrocytes has been demonstrated (13).

\section{DISCUSSION}

Distribution and expression of synemin. Previous investigations of avian synemin have shown it to be associated with the intermediate-filament subunits desmin and vimentin in muscle cells and erythrocytes $(12,13,15,32,42,50)$. In this report, we show that it is also associated with vimentin filaments in lens cells and that this association exhibits the same biochemical and ultrastructural characteristics as those in the other cell types.

Earlier studies raised the possibility that synemin might be expressed only in postmitotic, terminally differentiating cells, but the data presented here clearly demonstrate that this is not the case. Chicken embryo lens cells remain proliferative until ca. 4 days of incubation, when postmitotic fibers first begin to accumulate (17), and synemin is expressed throughout the lens before this stage. Similarly, in adult lens tissue, the epithelial cells anterior to the annular pad are presumably all capable of further division, and these cells possess synemin as well. Recent experiments have also demonstrated synemin in primitive-series erythroid cells (from 4.5-day embryos [unpublished data]), which have not yet undergone their terminal mitoses (3). The status of synemin in mitotic cells has not yet been determined.

Many factors are known to influence the growth and differentiation of lens cells. Inductive events occur during specific periods of embryogenesis, and different lens-specific proteins appear at different stages of differentiation of the cells as well as at different ages of the organism $(1,18,31,39$, 40). For example, the different crystallin polypeptides are not coordinately expressed, and they exhibit distinct embryonic and adult forms (56). Lens cells undergo limited differentiation in culture, and certain non-lens cells are capable of undergoing transdifferentiation to lentoid bodies that express lens-specific proteins (see, e.g., reference 33). We have investigated the appearance and accumulation of synemin in developing lens cells by using immunofluorescence and have noted a correlation of $: y$ nemin accumulation with cell elongation. The inductive event or influence that triggers this accumulation, however, has not been ascertained. Elucidation of factors that regulate the transcription of the synemin gene(s) and the stability and translatability of synemin mRNA, using both in vivo and in vitro approaches, may provide new clues about the regulation of expression of synemin and the role that synemin consequently fulfills in these cells.

In addition to avian lens cells, muscle cells, and erythrocytes, the only other cell type in which synemin has been found in relative abundance is the ependymal tanycyte (unpublished data). Immunofluorescence has revealed that synemin is especially abundant in the cell processes that extend radially from the central canal to the dorsal and ventral fissures of the chicken spinal cord. Synemin appears here very early in embryogenesis, in a small group of cells on the ventral side of the neural tube. Two-dimensional immunoautoradiography of spinal cord cytoskeletons has confirmed the identity of this antigen as synemin. The tanycyte processes are also packed with vimentin filaments but possess no detectable glial-filament protein, neurofilament proteins, or desmin, as assayed by immunofluorescence of certain developmental stages (however, see reference 49 for evidence of transient glial-filament protein expression by these cells in humans). These findings are thus consistent with the concepts of synemin existing in a very limited number of cell types, always with either vimentin or desmin, and existing in both proliferative and terminally differentiated cells. The basis of the common need for synemin in these particular cell types, however, remains to be determined.

Potential roles for synemin. Intermediate filaments are usually thought to be involved in cell structure (reviewed in reference 24), but substantiation of such a role has been elusive. They may provide form, volume, elasticity, and stability to lens cells and other cells in which they are abundant; perhaps this is a general role that some cell types, such as striated-muscle cells, have expanded upon to fulfill more specific structural needs (24). Associated proteins such as synemin would presumably be involved in such roles and would thus be expressed in a cell type-specific manner, as is observed.

Intermediate filaments can be found throughout the cytoplasmic domain, often in association with the plasma membrane. Evidence for the anchorage of intermediate filaments to plasma membranes comes primarily through microscopy; in some cases, specialized structures are involved, such as desmosomes for keratin filaments in epithelial cells $(6,51)$, dense bodies for desmin filaments in smooth-muscle cells $(5$, 53), and desmosomes and intercalated discs for desmin filaments in cardiac-muscle cells $(7,21,26)$. The only cases where specialized structures are not evident involve the anchorage of vimentin filaments to erythrocyte $(13,15)$ and lens $(2,45)$ plasma membranes and of desmin and vimentin filaments to skeletal-muscle plasma membranes $(11,26,41)$. It is of interest that these particular membranes are all lined with the protein spectrin $(35,48)$ and that the intermediate filaments anchored to them are all associated with synemin $(12,13,15$, this study). We are currently investigating the functional significance of this coincidence and hope to determine the involvement of both synemin and spectrin in filament-membrane interactions in these cells.

We previously proposed an intermediate-filament crosslinking function for synemin based on immunoelectronmicroscopic images of antibody-decorated erythrocyte filaments (13), thin sections showing cross bridges between 
intermediate filaments of cultured lens cells (46), the apparent self association of synemin in vitro (50) and by analogy to the large neurofilament-associated protein, for which a similar function had been proposed (55). The imunoelectronmicroscopic images of synemin in chicken lens cells, obtained in this study, are consistent with this interpretation. The possibility that the observed filament bridging is an artifact of bivalent antibodies was previously discounted on the basis of its insensitivity to formaldehyde fixation before decoration. This fixation was assumed to have immobilized the filaments on the substrate and precluded the possibility of artificially induced cross bridging; subsequent trials with glutaraldehyde under conditions that rendered the filaments insoluble in $6 \mathrm{M}$ urea but which did not destroy the antigenicity of synemin gave the same result (data not shown). However, the possibility of limited filament mobility after fixation cannot be ruled out by these experiments.

Attempts to resolve this issue by using Fab fragments of the synemin antibodies have been inconclusive. Highly purified Fab fragments bound the intermediate filaments of muscle cells and erythrocytes, as assayed by immunofluorescence, but periodic decoration of the filaments was not evident in electron micrographs. Amplification with Fab fragments of goat anti-rabbit immunoglobulin $\mathrm{G}$ similarly did not reveal any obvious periodic decoration. We have thus been unable to visualize synemin along the filaments with univalent antibodies. Since synemin is not evident as an appendage of these filaments unless bivalent antibodies are applied, it is conceivable that bivalent antibodies cause a conformational change in synemin upon binding. If synemin exhibits repeated epitopes, for example, the antibodies might ravel up each synemin polypeptide on a filament and make successive synemins appear widely separated, whereas these synemins would otherwise lie flat against the core filament in an extended, head-to-tail manner (see ref. 13 for alternative possibilities). Such raveling would imply that most of the synemin polypeptide is loosely associated with the core polymer, although some portion must simultaneously be tightly associated (perhaps a copolymerizing domain). Such an arrangement might permit synemin to project laterally from the core filament under certain conditions, to interact with other cellular structures.

Although we have no evidence to the contrary, whether synemin itself cross-links vimentin or desmin filaments in vivo remains to be conclusively demonstrated through nonserological approaches. The immunoelectron-microscopic images of synemin in lens cells are virtually indistinguishable from those of synemin in erythrocytes, however, and are certainly consistent with a cross-linking role, subject to the caveats outlined above. Other possible functions of synemin include stabilization of the filaments, linkage of the filaments to other cellular organelles, and perhaps even prevention of unwanted interactions. Lens cells, muscle cells, tanycytes, and erythrocytes all exhibit extensive arrays or networks of intermediate filaments, and the organization, maintenance, and regulation of these systems are likely to involve a complex set of interactions that undoubtedly include synemin.

\section{ACKNOWLEDGMENTS}

We thank John Ngai for his comments on the manuscript.

This work was supported by grants from the National Institutes of Health, National Science Foundation, and Muscular Dystrophy Association of America. B.L.G. was supported by a postdoctoral fellowship from the Muscular Dystrophy Association of America.
E.L. is a recipient of a Public Health Service Research Career Development Award from the National Institutes of Health.

\section{LITERATURE CITED}

1. Bloemendal, H. 1977. The vertebrate eye lens. Science 197:127138.

2. Bradley, R. H., M. Ireland, and H. Maisel. 1979. The cytoskeleton of chick lens cells. Exp. Eye Res. 28:441-453.

3. Bruns, G. A. P., and V. M. Ingram. 1973. The erythroid cells and haemoglobins of the chick embryo. Phil. Trans. Roy. Soc. Lond. Ser. B 266:225-305.

4. Burridge, K. 1978. Direct indentification of specific glycoproteins and antigens in sodium dodecyl sulfate gels. Methods Enzymol. 50:54-64.

5. Cooke, P. H., and R. H. Chase. 1971. Potassium chlorideinsoluble myofilaments in vertebrate smooth muscle cells. Exp. Cell Res. 66:417-425.

6. Drochmans, P., C. Freudenstein, J.-C. Wanson, L. Laurent, T. W. Keenan, J. Stadler, R. Leloup, and W. W. Franke. 1978. Structure and biochemical composition of desmosomes and tonofilaments isolated from calf muzzle epidermis. J. Cell Biol. 79:427-443.

6a.Ellis, M., S. Alousi, J. Lawniczak, H. Maisel, and M. Welsh. 1984. Studies on lens vimentin. Exp. Eye Res. 38:195-202.

7. Ferrans, V. J., and W. C. Roberts. 1973. Intermyofibrillar and nuclear-myofibrillar connections in human and canine myocardium. An ultrastructural study. J. Mol. Cell. Cardiol. 5:247-257.

8. Garadi, R., M. Katar, and H. Maisel. 1983. Two-dimensional gel analysis of chick lens proteins. Exp. Eye Res. 36:859-869.

9. Gard, D. L., and E. Lazarides. 1982. Cyclic AMP-modulated phosphorylation of intermediate filament proteins in cultured avian myogenic cells. Mol. Cell. Biol. 2:1104-1114.

10. Gershoni, J. M., and G. E. Palade. 1983. Protein blotting: principles and applications. Anal. Biochem. 131:1-15.

11. Granger, B. L., and E. Lazarides. 1979. Desmin and vimentin coexist at the periphery of the myofibril $\mathrm{Z}$ disc. Cell 18:10531063.

12. Granger, B. L., and E. Lazarides. 1980. Synemin: a new high molecular weight protein associated with desmin and vimentin filaments in muscle. Cell 22:727-738.

13. Granger, B. L., and E. Lazarides. 1982. Structural associations of synemin and vimentin filaments in avian erythrocytes revealed by immunoelectron microscopy. Cell 30:263-275.

14. Granger, B. L., and E. Lazarides. 1983. Expression of the major neurofilament subunit in chicken erythrocytes. Science 221:553-556.

14a.Granger, B. L., and E. Lazarides. 1984. Membrane skeletal protein 4.1 of avian erythrocytes is composed of multiple variants that exhibit tissue-specific expression. Cell 37:595-607.

15. Granger, B. L., E. A. Repasky, and E. Lazarides. 1982. Synemin and vimentin are components of intermediate filaments in avian erythrocytes. J. Cell Biol. 92:299-312.

16. Hamburger, V., and H. L. Hamilton. 1951. A series of normal stages in the development of the chick embryo. J. Morphol. 88:49-92.

17. Hanna, C., and H. C. Keatts. 1966. Chicken lens development: epithelial cell production and migration. Exp. Eye Res. 5:111115.

18. Harding, J. J., and K. J. Dilley. 1976. Structural proteins of the mammalian lens: a review with emphasis on changes in development, aging and cataract. Exp. Eye Res. 22:1-73.

18a.Hatfield, J. S., R. P. Skoff, H. Maisel, and L. Eng. 1984. Glial fibrillary acidic protein is localized in the lens epithelium. J. Cell Biol. 98:1895-1898.

19. Ireland, M., and H. Maisel. 1983. Identification of native actin filaments in chick lens fiber cells. Exp. Eye Res. 36:531-536.

20. Johnson, G. D., R. S. Davidson, K. C. McNamee, G. Russell, D. Goodwin, and E. J. Holborow. 1982. Fading of immunofluorescence during microscopy: a study of the phenomenon and its remedy. J. Immunol. Meth. 55:231-242.

21. Kartenbeck, J., W. W. Franke, J. G. Moser, and U. Stoffels. 1983. Specific attachment of desmin filaments to desmosomal plaques in cardiac myocytes. EMBO J. 2:735-742. 
22. Kibbelaar, M. A., A.-M. E. Selten-Versteegen, I. Dunia, E. L. Benedetti, and H. Bloemendal. 1979. Actin in mammalian lens. Eur. J. Biochem. 95:543-549.

23. Laemmli, U. K. 1970. Cleavage of structural proteins during the assembly of the head of bacteriophage T4. Nature (London) 227:680-685

24. Lazarides, E. 1980. Intermediate filaments as mechanical integrators of cellular space. Nature (London) 283:249-256.

25. Lazarides, E., and B. L. Granger. 1982. Preparation and assay of the intermediate filament proteins desmin and vimentin. Methods Enzymol. 85:488-508.

26. Lazarides, E., and B. D. Hubbard. 1976. Immunological characterization of the subunit of the $100 \AA$ filaments from muscle cells. Proc. Natl. Acad. Sci. U.S.A. 73:4344-4348.

27. Lieska, N., J. Chen, H. Maisel, and A. E. Romero-Herrera. 1980. Subunit characterization of lens intermediate filaments. Biochem. Biophys. Acta 626:136-153.

28. Lo, W. K., and H. Maisel. 1979. Intermediate filament protein of chick lens epithelial cells. Ophthalmic Res. 11:129-135.

29. Maisel, H., N. Lieska, and R. Bradley. 1978. Isolation of filaments of the chick lens. Experientia 34:352-353.

30. Maisel, H., and M. M. Perry. 1972. Electron microscope observations on some structural proteins of the chick lens. Exp. Eye Res. 14:7-12.

31. McAvoy, J. W. 1980. Induction of the eye lens. Differentiation 17:137-149.

32. Moon, R. T., and E. Lazarides. 1983. Synthesis and posttranslational assembly of intermediate filaments in avian erythroid cells: vimentin assembly limits the rate of synemin assembly. Proc. Natl. Acad. Sci. U.S.A. 80:5495-5499.

33. Moscona, A. A., M. Brown, L. Degenstein, L. Fox, and B. M. Soh. 1983. Transformation of retinal glia cells into lens phenotype: expression of MP26, a lens plasma membrane antigen. Proc. Natl. Acad. Sci. U.S.A. 80:7239-7243.

34. Nelson, W. J., B. L. Granger, and E. Lazarides. 1983. Avian lens spectrin: subunit composition compared with erythrocyte and brain spectrin. J. Cell Biol. 97:1271-1276.

35. Nelson, W. J., and E. Lazarides. 1983. Expression of the $\beta$ subunit of spectrin in nonerythroid cells. Proc. Natl. Acad. Sci. U.S.A. 80:363-367.

36. O'Connor, C. M., D. R. Balzer, and E. Lazarides. 1979. Phosphorylation of subunit proteins of intermediate filaments from chicken muscle and nonmuscle cells. Proc. Natl. Acad. Sci. U.S.A. 76:819-823.

37. O'Farrell, P. H. 1975. High resolution two-dimensional electrophoresis of proteins. J. Biol. Chem. 250:4007-4021.

38. O'Rahilly, R., and D. B. Meyer. 1959. The early development of the eye in the chick Gallus domesticus (stages 8 to 25). Acta Anat. 36:20-58.

39. Papaconstantinou, J. 1967. Molecular aspects of lens cell differentiation. Science 156:338-346.

40. Piatigorsky, J. 1981. Lens differentiation in vertebrates. A review of cellular and molecular features. Differentiation
19:134-153.

41. Pierobon-Bormioli, S. 1981. Transverse sarcomere filamentous systems: "Z- and M-cables." J. Muscle Res. Cell Motil. 2:401413.

42. Price, M. G., and E. Lazarides. 1983. Expression of intermediate filament-associated proteins paranemin and synemin in chicken development. J. Cell Biol. 97:1860-1874.

43. Rafiferty, N. S., and W. Goossens. 1978. Cytoplasmic filaments in the crystalline lens of various species: functional correlations. Exp. Eye Res. 26:177-190.

44. Ramaekers, F. C. S., T. R. Boomkens, and H. Bloemendal. 1981. Cytoskeletal and contractile structures in bovine lens cell differentiation. Exp. Cell Res. 135:454-461.

45. Ramaekers, F. C. S., I. Dunia, H. J. Dodemont, E. L. Benedetti, and H. Bloemendal. 1982. Lenticular intermediate-sized filaments: biosynthesis and interaction with plasma membrane. Proc. Natl. Acad. Sci. U.S.A. 79:3208-3212.

46. Ramaekers, F. C. S., M. Osborn, E. Schmid, K. Weber, H. Bloemendal, and W. W. Franke. 1980. Identification of the cytoskeletal proteins in lens-forming cells, a special epitheloid cell type. Exp. Cell Res. 127:309-327.

47. Ramaekers, F. C. S., L. G. Poels, P. H. K. Jap, and H. Bloemendal. 1982. Simultaneous demonstration of microfilaments and intermediate-sized filaments in the lens by double immunofluorescence. Exp. Eye Res. 35:363-369.

48. Repasky, E. A., B. L. Granger, and E. Lazarides. 1982. Widespread occurrence of avian spectrin in non-erythroid cells. Cell 29:821-833.

49. Roessmann, U., M. E. Velasco, S. D. Sindely, and P. Gambetti. 1980. Glial fibrillary acidic protein (GFAP) in ependymal cells during development. An immunocytochemical study. Brain Res. 200:13-21.

50. Sandoval, I. V., C. A. L. S. Colaco, and E. Lazarides. 1983. Purification of the intermediate filament-associated protein, synemin, from chicken smooth muscle. Studies on its physicochemical properties, interaction with desmin, and phosphorylation. J. Biol. Chem. 258:2568-2576.

51. Staehelin, L. A. 1974. Structure and function of intercellular junctions. Int. Rev. Cytol. 39:191-283.

52. Towbin, H., T. Staehelin, and J. Gordon. 1979. Electrophoretic transfer of proteins from polyacrylamide gels to nitrocellulose sheets: procedure and some applications. Proc. Natl. Acad. Sci. U.S.A. 76:4350-4354.

53. Uehara, Y., G. R. Campbell, and G. Burnstock. 1971. Cytoplasmic filaments in developing and adult vertebrate smooth muscle. J. Cell Biol. 50:484-497.

54. Wang, C., R. H. Gomer, and E. Lazarides. 1981. Heat shock proteins are methylated in avian and mammalian cells. Proc. Natl. Acad. Sci. U.S.A. 78:3531-3535.

55. Willard, M., and C. Simon. 1981. Antibody decoration of neurofilaments. J. Cell Biol. 89:198-205.

56. Zwaan, J., and A. Ikeda. 1968. Macromolecular events during differentiation of the chicken lens. Exp. Eye Res. 7:301-311. 